\title{
Sharp Bounds for Neuman Means by Harmonic, Arithmetic, and Contraharmonic Means
}

\author{
Zhi-Jun Guo, ${ }^{1}$ Yu-Ming Chu, ${ }^{1}$ Ying-Qing Song, ${ }^{1}$ and Xiao-Jing Tao ${ }^{2}$ \\ ${ }^{1}$ School of Mathematics and Computation Sciences, Hunan City University, Yiyang 413000, China \\ ${ }^{2}$ College of Mathematics and Econometrics, Hunan University, Changsha 410082, China
}

Correspondence should be addressed to Yu-Ming Chu; chuyuming2005@126.com and Ying-Qing Song; 1452225875@qq.com

Received 20 May 2014; Accepted 12 July 2014; Published 23 July 2014

Academic Editor: Shuangjie Peng

Copyright (C) 2014 Zhi-Jun Guo et al. This is an open access article distributed under the Creative Commons Attribution License, which permits unrestricted use, distribution, and reproduction in any medium, provided the original work is properly cited.

We give several sharp bounds for the Neuman means $N_{A H}$ and $N_{H A}\left(N_{C A}\right.$ and $\left.N_{A C}\right)$ in terms of harmonic mean $H$ (contraharmonic mean $C$ ) or the geometric convex combination of arithmetic mean $A$ and harmonic mean $H$ (contraharmonic mean $C$ and arithmetic mean $A$ ) and present a new chain of inequalities for certain bivariate means.

\section{Introduction}

For $a, b>0$ with $a \neq b$, the Schwab-Borchardt mean $\operatorname{SB}(a, b)$ $[1-3]$ of $a$ and $b$ is defined as

$$
\operatorname{SB}(a, b)= \begin{cases}\frac{\sqrt{b^{2}-a^{2}}}{\cos ^{-1}(a / b)}, & a<b, \\ \frac{\sqrt{a^{2}-b^{2}}}{\cosh ^{-1}(a / b)}, & a>b,\end{cases}
$$

where $\cos ^{-1}(x)$ and $\cosh ^{-1}(x)=\log \left(x+\sqrt{x^{2}-1}\right)$ are the inverse cosine and inverse hyperbolic cosine functions, respectively.

The Schwab-Borchardt mean $\operatorname{SB}(a, b)$ can be expressed by the symmetric elliptic integral $R_{F}[4]$ of the first kind as follows [5] (see also [6, (3.21)]):

$$
\operatorname{SB}(a, b)=\frac{1}{R_{F}\left(a^{2}, b^{2}, b^{2}\right)},
$$

where $R_{F}(a, b, c)=(1 / 2) \int_{0}^{\infty}[(t+a)(t+b)(t+c)]^{-1 / 2} d t$.

Recently, the Schwab-Borchardt mean has been the subject of intensive research. In particular, many remarkable inequalities for the Schwab-Borchardt mean and its generated means can be found in the literature [1-3, 7-10].
Very recently, Neuman [11] found a new mean $N(a, b)$ derived from the Schwab-Borchardt mean as follows:

$$
N(a, b)=\frac{1}{2}\left[a+\frac{b^{2}}{\mathrm{SB}(a, b)}\right] .
$$

Let $G(a, b)=\sqrt{a b}, H(a, b)=2 a b /(a+b), L(a, b)=$ $(a-b) /(\log a-\log b), P(a, b)=(a-b) /\left[2 \sin ^{-1}((a-b) /(a+\right.$ $b))], A(a, b)=(a+b) / 2, M(a, b)=(a-b) /\left[2 \sinh ^{-1}((a-\right.$ b) $/(a+b))], T(a, b)=(a-b) /\left[2 \tan ^{-1}((a-b) /(a+b))\right]$, $Q(a, b)=\sqrt{\left(a^{2}+b^{2}\right) / 2}$, and $C(a, b)=\left(a^{2}+b^{2}\right) /(a+$ b) be, respectively, the geometric, harmonic, logarithmic, first Seiffert, arithmetic, Neuman-Sándor, second Seiffert, quadratic, and contraharmonic means, and let

$$
\begin{aligned}
& N_{A H}(a, b)=N[A(a, b), H(a, b)], \\
& N_{H A}(a, b)=N[H(a, b), A(a, b)], \\
& N_{A G}(a, b)=N[A(a, b), G(a, b)], \\
& N_{G A}(a, b)=N[G(a, b), A(a, b)], \\
& N_{A C}(a, b)=N[A(a, b), C(a, b)], \\
& N_{C A}(a, b)=N[C(a, b), A(a, b)], \\
& N_{A Q}(a, b)=N[A(a, b), Q(a, b)], \\
& N_{Q A}(a, b)=N[Q(a, b), A(a, b)]
\end{aligned}
$$


be the Neuman means. Then Neuman [11] proved that

$$
\begin{aligned}
G(a, b) & <L(a, b)<N_{A G}(a, b)<P(a, b)<N_{G A}(a, b) \\
& <A(a, b)<M(a, b)<N_{\mathrm{QA}}(a, b)<T(a, b) \\
& <N_{A Q}(a, b)<Q(a, b)<C(a, b),
\end{aligned}
$$

for all $a, b>0$ with $a \neq b$, and the double inequalities

$$
\begin{aligned}
& \alpha_{1} A(a, b)+\left(1-\alpha_{1}\right) G(a, b) \\
& \quad<N_{G A}(a, b)<\beta_{1} A(a, b)+\left(1-\beta_{1}\right) G(a, b), \\
& \alpha_{2} Q(a, b)+\left(1-\alpha_{2}\right) A(a, b) \\
& \quad<N_{A Q}(a, b)<\beta_{2} Q(a, b)+\left(1-\beta_{2}\right) A(a, b), \\
& \alpha_{3} A(a, b)+\left(1-\alpha_{3}\right) G(a, b) \\
& \quad<N_{A G}(a, b)<\beta_{3} A(a, b)+\left(1-\beta_{3}\right) G(a, b), \\
& \alpha_{4} Q(a, b)+\left(1-\alpha_{4}\right) A(a, b) \\
& \quad<N_{Q A}(a, b)<\beta_{4} Q(a, b)+\left(1-\beta_{4}\right) A(a, b)
\end{aligned}
$$

hold for all $a, b>0$ with $a \neq b$ if and only if $\alpha_{1} \leq 2 / 3, \beta_{1} \geq$ $\pi / 4, \alpha_{2} \leq 2 / 3, \beta_{2} \geq(\pi-2) /[4(\sqrt{2}-1)]=0.689 \ldots, \alpha_{3} \leq 1 / 3$, $\beta_{3} \geq 1 / 2, \alpha_{4} \leq 1 / 3$, and $\beta_{4} \geq[\log (1+\sqrt{2})+\sqrt{2}-2] /[2(\sqrt{2}-$ $1)]=0.356 \ldots$

Zhang et al. [12] presented the best possible parameters $\alpha_{1}, \alpha_{2}, \beta_{1}, \beta_{2} \in[0,1 / 2]$ and $\alpha_{3}, \alpha_{4}, \beta_{3}, \beta_{4} \in[1 / 2,1]$ such that the double inequalities

$$
\begin{aligned}
& G\left(\alpha_{1} a+\left(1-\alpha_{1}\right) b, \alpha_{1} b+\left(1-\alpha_{1}\right) a\right) \\
& \quad<N_{A G}(a, b)<G\left(\beta_{1} a+\left(1-\beta_{1}\right) b, \beta_{1} b+\left(1-\beta_{1}\right) a\right), \\
& G\left(\alpha_{2} a+\left(1-\alpha_{2}\right) b, \alpha_{2} b+\left(1-\alpha_{2}\right) a\right) \\
& \quad<N_{G A}(a, b)<G\left(\beta_{2} a+\left(1-\beta_{2}\right) b, \beta_{2} b+\left(1-\beta_{2}\right) a\right), \\
& Q\left(\alpha_{3} a+\left(1-\alpha_{3}\right) b, \alpha_{3} b+\left(1-\alpha_{3}\right) a\right) \\
& \quad<N_{\mathrm{QA}}(a, b)<Q\left(\beta_{3} a+\left(1-\beta_{3}\right) b, \beta_{3} b+\left(1-\beta_{3}\right) a\right), \\
& Q\left(\alpha_{4} a+\left(1-\alpha_{4}\right) b, \alpha_{4} b+\left(1-\alpha_{4}\right) a\right) \\
& \quad<N_{A Q}(a, b)<Q\left(\beta_{4} a+\left(1-\beta_{4}\right) b, \beta_{4} b+\left(1-\beta_{4}\right) a\right)
\end{aligned}
$$

hold for all $a, b>0$ with $a \neq b$.

In [13], the authors found the greatest values $\alpha_{1}, \alpha_{2}, \alpha_{3}$, $\alpha_{4}, \alpha_{5}, \alpha_{6}, \alpha_{7}$, and $\alpha_{8}$ and the least values $\beta_{1}, \beta_{2}, \beta_{3}, \beta_{4}, \beta_{5}, \beta_{6}$, $\beta_{7}$, and $\beta_{8}$ such that the double inequalities

$$
\begin{aligned}
& A^{\alpha_{1}}(a, b) G^{1-\alpha_{1}}(a, b) \\
& \quad<N_{G A}(a, b)<A^{\beta_{1}}(a, b) G^{1-\beta_{1}}(a, b), \\
& \frac{\alpha_{2}}{G(a, b)}+\frac{1-\alpha_{2}}{A(a, b)} \\
& \quad<\frac{1}{N_{G A}(a, b)}<\frac{\beta_{2}}{G(a, b)}+\frac{1-\beta_{2}}{A(a, b)},
\end{aligned}
$$

$$
\begin{aligned}
& A^{\alpha_{3}}(a, b) G^{1-\alpha_{3}}(a, b) \\
& <N_{A G}(a, b)<A^{\beta_{3}}(a, b) G^{1-\beta_{3}}(a, b), \\
& \frac{\alpha_{4}}{G(a, b)}+\frac{1-\alpha_{4}}{A(a, b)} \\
& \quad<\frac{1}{N_{A G}(a, b)}<\frac{\beta_{4}}{G(a, b)}+\frac{1-\beta_{4}}{A(a, b)}, \\
& Q^{\alpha_{5}}(a, b) A^{1-\alpha_{5}}(a, b) \\
& \quad<N_{A Q}(a, b)<Q^{\beta_{5}}(a, b) A^{1-\beta_{5}}(a, b), \\
& \frac{\alpha_{6}}{A(a, b)}+\frac{1-\alpha_{6}}{Q(a, b)} \\
& \quad<\frac{1}{N_{A Q}(a, b)}<\frac{\beta_{6}}{A(a, b)}+\frac{1-\beta_{6}}{Q(a, b)}, \\
& Q^{\alpha_{7}}(a, b) A^{1-\alpha_{7}}(a, b) \\
& <N_{\mathrm{QA}}(a, b)<Q^{\beta_{7}}(a, b) A^{1-\beta_{7}}(a, b), \\
& \frac{\alpha_{8}}{A(a, b)}+\frac{1-\alpha_{8}}{Q(a, b)} \\
& \quad<\frac{\beta_{8}}{A(a, b)}+\frac{1-\beta_{8}}{Q(a, b)}
\end{aligned}
$$

hold for all $a, b>0$ with $a \neq b$.

Let $a>b>0, v=(a-b) /(a+b) \in(0,1), p \in(0, \infty)$, $q \in(0, \pi / 2), r \in(0, \log (2+\sqrt{3}))$, and $s \in(0, \pi / 3)$ be the parameters such that $1 / \cosh (p)=\cos (q)=1-v^{2}, \cosh (r)=$ $\sec (s)=1+v^{2}$. Then He et al. [14] proved that

$$
\begin{gathered}
N_{A H}(a, b)=\frac{1}{2} A(a, b)\left[1+\frac{2 p}{\sinh (2 p)}\right], \\
N_{H A}(a, b)=\frac{1}{2} A(a, b)\left[\cos (q)+\frac{q}{\sin (q)}\right], \\
N_{C A}(a, b)=\frac{1}{2} A(a, b)\left[\cosh (r)+\frac{r}{\sinh (r)}\right], \\
N_{A C}(a, b)=\frac{1}{2} A(a, b)\left[1+\frac{2 s}{\sin (2 s)}\right], \\
H(a, b)<N_{A H}(a, b)<N_{H A}(a, b)<A(a, b) \\
<N_{C A}(a, b)<N_{A C}(a, b)<C(a, b) .
\end{gathered}
$$

Let $x \in[0,1 / 2], y \in[1 / 2,1], f(x)=H[x a+(1-x) b, x b+$ $(1-x) a]$, and $g(y)=C[y a+(1-y) b, y b+(1-y) a]$. Then we clearly see that 


$$
\begin{aligned}
f(0) & =H(a, b)<N_{A H}(a, b)<N_{H A}(a, b)<A(a, b) \\
& =f\left(\frac{1}{2}\right)=g\left(\frac{1}{2}\right)<N_{C A}(a, b)<N_{A C}(a, b)<C(a, b) \\
& =g(1),
\end{aligned}
$$

for all $a, b>0$ with $a \neq b$, and the functions $f$ and $g$ are, respectively, strictly increasing on the intervals $[0,1 / 2]$ and $[1 / 2,1]$ for fixed $a, b>0$ with $a \neq b$.

The main purpose of this paper is to find the best possible parameters $\alpha_{1}, \alpha_{2}, \alpha_{3}, \alpha_{4}, \beta_{1}, \beta_{2}, \beta_{3}, \beta_{4} \in \mathbb{R}, \alpha_{5}, \alpha_{6}, \beta_{5}, \beta_{6} \in$ $[0,1 / 2]$, and $\alpha_{7}, \alpha_{8}, \beta_{7}, \beta_{8} \in[1 / 2,1]$ such that the double inequalities

$$
\begin{aligned}
& A^{\alpha_{1}}(a, b) H^{1-\alpha_{1}}(a, b) \\
& \quad<N_{A H}(a, b)<A^{\beta_{1}}(a, b) H^{1-\beta_{1}}(a, b), \\
& A^{\alpha_{2}}(a, b) H^{1-\alpha_{2}}(a, b) \\
& \quad<N_{H A}(a, b)<A^{\beta_{2}}(a, b) H^{1-\beta_{2}}(a, b), \\
& C^{\alpha_{3}}(a, b) A^{1-\alpha_{3}}(a, b) \\
& \quad<N_{C A}(a, b)<C^{\beta_{3}}(a, b) A^{1-\beta_{3}}(a, b), \\
& C^{\alpha_{4}}(a, b) A^{1-\alpha_{4}}(a, b) \\
& \quad<N_{A C}(a, b)<C^{\beta_{4}}(a, b) A^{1-\beta_{4}}(a, b), \\
& H\left[\alpha_{5} a+\left(1-\alpha_{5}\right) b, \alpha_{5} b+\left(1-\alpha_{5}\right) a\right] \\
& \quad<N_{A H}(a, b)<H\left[\beta_{5} a+\left(1-\beta_{5}\right) b, \beta_{5} b+\left(1-\beta_{5}\right) a\right], \\
& H\left[\alpha_{6} a+\left(1-\alpha_{6}\right) b, \alpha_{6} b+\left(1-\alpha_{6}\right) a\right] \\
& \quad<N_{H A}(a, b)<H\left[\beta_{6} a+\left(1-\beta_{6}\right) b, \beta_{6} b+\left(1-\beta_{6}\right) a\right], \\
& C\left[\alpha_{7} a+\left(1-\alpha_{7}\right) b, \alpha_{7} b+\left(1-\alpha_{7}\right) a\right] \\
& \quad<N_{C A}(a, b)<H\left[\beta_{7} a+\left(1-\beta_{7}\right) b, \beta_{7} b+\left(1-\beta_{7}\right) a\right], \\
& \quad\left[\alpha_{8} a+\left(1-\alpha_{8}\right) b, \alpha_{8} b+\left(1-\alpha_{8}\right) a\right] \\
& \quad(a, b)<H\left[\beta_{8} a+\left(1-\beta_{8}\right) b, \beta_{8} b+\left(1-\beta_{8}\right) a\right]
\end{aligned}
$$

hold, for all $a, b>0$ with $a \neq b$, and present a new chain of inequalities for certain bivariate means.

\section{Lemmas}

In order to prove our main results we need several lemmas, which we present in this section.

Lemma 1 (see [15, Theorem 1.25]). For $-\infty<a<b<\infty$, let $f, g:[a, b] \rightarrow \mathbb{R}$ be continuous on $[a, b]$ and differentiable on $(a, b)$; let $g^{\prime}(x) \neq 0$ on $(a, b)$. If $f^{\prime}(x) / g^{\prime}(x)$ is increasing (decreasing) on $(a, b)$, then so are

$$
\frac{f(x)-f(a)}{g(x)-g(a)}, \quad \frac{f(x)-f(b)}{g(x)-g(b)} .
$$

If $f^{\prime}(x) / g^{\prime}(x)$ is strictly monotone, then the monotonicity in the conclusion is also strict.

Lemma 2 (see [16, Lemma 1.1]). Suppose that the power series $f(x)=\sum_{n=0}^{\infty} a_{n} x^{n}$ and $g(x)=\sum_{n=0}^{\infty} b_{n} x^{n}$ have the radius of convergence $r>0$ and $a_{n}, b_{n}>0$ for all $n \geq 0$. If the sequence $\left\{a_{n} / b_{n}\right\}$ is (strictly) increasing (decreasing) for all $n \geq 0$, then the function $f(x) / g(x)$ is also (strictly) increasing (decreasing) on $(0, r)$.

Lemma 3. The function

$$
\varphi_{1}(x)=\frac{\sinh (x)-x}{\sinh (x)[\cosh (x)-1]}
$$

is strictly decreasing from $(0, \infty)$ onto $(0,1 / 3)$.

Proof. Making use of power series expansion we get

$$
\begin{aligned}
\varphi_{1}(x) & =\frac{2 \sinh (x)-2 x}{\sinh (2 x)-2 \sinh (x)} \\
& =\frac{\sum_{n=1}^{\infty}(2 /(2 n+1) !) x^{2 n+1}}{\sum_{n=1}^{\infty}\left(\left(2^{2 n+1}-2\right) /(2 n+1) !\right) x^{2 n+1}} \\
& =\frac{\sum_{n=0}^{\infty}(2 /(2 n+3) !) x^{2 n}}{\sum_{n=0}^{\infty}\left(\left(2^{2 n+3}-2\right) /(2 n+3) !\right) x^{2 n}} .
\end{aligned}
$$

Let

$$
a_{n}=\frac{2}{(2 n+3) !}, \quad b_{n}=\frac{2^{2 n+3}-2}{(2 n+3) !} .
$$

Then

$$
a_{n}>0, \quad b_{n}>0
$$

and $a_{n} / b_{n}=1 /\left(2^{2 n+2}-1\right)$ is strictly decreasing for all $n \geq 0$.

Note that

$$
\varphi_{1}\left(0^{+}\right)=\frac{a_{0}}{b_{0}}=\frac{1}{3}, \quad \varphi_{1}(\infty)=\lim _{n \rightarrow \infty} \frac{a_{n}}{b_{n}}=0 .
$$

Therefore, Lemma 3 follows easily from Lemma 2 and (18)-(21) together with the monotonicity of the sequence $\left\{a_{n} / b_{n}\right\}$.

Lemma 4. The function

$$
\varphi_{2}(x)=\frac{x-\sin (x)}{\sin (x)[1-\cos (x)]}
$$

is strictly increasing from $(0, \pi / 2)$ onto $(1 / 3, \pi / 2-1)$. 
Proof. Let $f_{1}(x)=x-\sin (x)$ and $g_{1}(x)=\sin (x)[1-\cos (x)]$. Then simple computations lead to

$$
\varphi_{2}(x)=\frac{f_{1}(x)}{g_{1}(x)}=\frac{f_{1}(x)-f_{1}(0)}{g_{1}(x)-g_{1}(0)},
$$

and $f_{1}^{\prime}(x) / g_{1}^{\prime}(x)=1 /[1+2 \cos (x)]$ is strictly increasing on $(0, \pi / 2)$

Note that

$$
\varphi_{2}\left(0^{+}\right)=\lim _{x \rightarrow 0^{+}} \frac{f_{1}^{\prime}(x)}{g_{1}^{\prime}(x)}=\frac{1}{3}, \quad \varphi_{2}\left(\frac{\pi}{2}\right)=\frac{\pi}{2}-1 .
$$

Therefore, Lemma 4 follows from Lemma 1, (23), (24), and the monotonicity of $f_{1}^{\prime}(x) / g_{1}^{\prime}(x)$.

Lemma 5. The function

$$
\varphi_{3}(x)=\frac{\log [(x+\sinh (x) \cosh (x)) /(2 \sinh (x) \cosh (x))]}{\log (1 / \cosh (x))}
$$

is strictly decreasing from $(0, \infty)$ onto $(0,2 / 3)$.

Proof. Let $f_{2}(x)=\log [(x+\sinh (x) \cosh (x)) /(2 \sinh (x)$ $\cosh (x))]$ and $g_{2}(x)=\log [1 / \cosh (x)]$. Then simple computations lead to

$$
\varphi_{3}(x)=\frac{f_{2}(x)}{g_{2}(x)}=\frac{f_{2}(x)-f_{2}\left(0^{+}\right)}{g_{2}(x)-g_{2}(0)}
$$

$$
\begin{aligned}
& \frac{f_{2}^{\prime}(x)}{g_{2}^{\prime}(x)} \\
& =\left(x\left[\cosh ^{3}(x)+\sinh ^{2}(x) \cosh (x)\right]-\sinh (x) \cosh ^{2}(x)\right) \\
& \quad \times\left(\sinh ^{2}(x) \cosh (x)[x+\sinh (x) \cosh (x)]\right)^{-1} \\
& =(8 x \cosh (3 x)+8 x \cosh (x)-4 \sinh (3 x)-4 \sinh (x)) \\
& \quad \times(4 x \cosh (3 x)-4 x \cosh (x)+\sinh (5 x)-\sinh (3 x) \\
& \quad-2 \sinh (x))^{-1} \\
& =\left(\sum _ { n = 1 } ^ { \infty } \left(\left(8(2 n+1)\left(1+3^{2 n}\right)-4\left(1+3^{2 n+1}\right)\right)\right.\right. \\
& \left.\left.\quad \times((2 n+1) !)^{-1}\right) x^{2 n+1}\right) \\
& \quad \times\left(\sum _ { n = 1 } ^ { \infty } \left(\left(4(2 n+1)\left(3^{2 n}-1\right)+5^{2 n+1}-3^{2 n+1}-2\right)\right.\right. \\
& \left.\left.\quad \times((2 n+1) !)^{-1}\right) x^{2 n+1}\right)^{-1}
\end{aligned}
$$

$$
\begin{aligned}
=\left(\sum_{n=0}^{\infty}(\right. & \left(8(2 n+3)\left(1+3^{2 n+2}\right)-4\left(1+3^{2 n+3}\right)\right) \\
& \left.\left.\times((2 n+3) !)^{-1}\right) x^{2 n}\right) \\
\times\left(\sum_{n=0}^{\infty}(\right. & \left(4(2 n+3)\left(3^{2 n+2}-1\right)+5^{2 n+3}-3^{2 n+3}-2\right) \\
& \left.\left.\times((2 n+3) !)^{-1}\right) x^{2 n}\right)^{-1} .
\end{aligned}
$$

Let

$$
\begin{gathered}
a_{n}=\frac{8(2 n+3)\left(1+3^{2 n+2}\right)-4\left(1+3^{2 n+3}\right)}{(2 n+3) !}, \\
b_{n}=\frac{4(2 n+3)\left(3^{2 n+2}-1\right)+5^{2 n+3}-3^{2 n+3}-2}{(2 n+3) !} .
\end{gathered}
$$

Then it is not difficult to verify that

$$
\begin{gathered}
a_{n}=\frac{(16 n+20)+(16 n+12) 3^{2 n+2}}{(2 n+3) !}>0, \quad b_{n}>0, \\
\frac{a_{n+1}}{b_{n+1}}-\frac{a_{n}}{b_{n}}=-\frac{c_{n}}{d_{n}}<0
\end{gathered}
$$

for all $n \geq 0$, where $c_{n}=(256 n+48) 3^{2 n+2} \times 5^{2 n+3}+(384 n+$ $464) 5^{2 n+3}+\left(2048 n^{2}+7040 n+6000\right) 3^{2 n+2}-16 \cdot 3^{4 n+7}+64$ and $d_{n}=\left[5^{2 n+5}+(8 n+17) 3^{2 n+4}-(8 n+22)\right]\left[5^{2 n+3}+(8 n+9) 3^{2 n+2}-\right.$ $(8 n+14)]$.

Note that

$$
\varphi_{3}\left(0^{+}\right)=\frac{a_{0}}{b_{0}}=\frac{2}{3}, \quad \varphi_{3}(\infty)=0 .
$$

It follows from Lemma 2 and (27)-(29) that the function $f_{2}^{\prime}(x) / g_{2}^{\prime}(x)$ is strictly decreasing on $(0, \infty)$. Therefore, Lemma 5 follows from (26) and (30) together with Lemma 1 and the monotonicity of $f_{2}^{\prime}(x) / g_{2}^{\prime}(x)$.

Lemma 6. The function

$$
\varphi_{4}(x)=\frac{\log [(x+\sin (x) \cos (x)) /(2 \sin (x))]}{\log [\cos (x)]}
$$

is strictly decreasing from $(0, \pi / 2)$ onto $(0,1 / 3)$.

Proof. Let $f_{3}(x)=\log [(x+\sin (x) \cos (x)) /(2 \sin (x))], g_{3}(x)=$ $\log [\cos (x)], f_{4}(x)=\cos ^{2}(x)[x-\sin (x) \cos (x)]$, and $g_{4}(x)=$ $\sin ^{2}(x)[x+\sin (x) \cos (x)]$. Then simple computations lead to

$$
\begin{aligned}
& \varphi_{4}(x)=\frac{f_{3}(x)}{g_{3}(x)}=\frac{f_{3}(x)-f_{3}\left(0^{+}\right)}{g_{3}(x)-g_{3}(0)}, \\
& \frac{f_{3}^{\prime}(x)}{g_{3}^{\prime}(x)}=\frac{f_{4}(x)}{g_{4}(x)}=\frac{f_{4}(x)-f_{4}(0)}{g_{4}(x)-g_{4}(0)}, \\
& \frac{f_{4}^{\prime}(x)}{g_{4}^{\prime}(x)}=1-\frac{1}{1 / 2+\sin (2 x) /(2 x)} .
\end{aligned}
$$


Since the function $x \rightarrow \sin (x) / x$ is strictly decreasing on $(0, \pi)$, hence (34) leads to the conclusion that $f_{4}^{\prime}(x) / g_{4}^{\prime}(x)$ is strictly decreasing on $(0, \pi / 2)$.

Note that

$$
\varphi_{4}\left(0^{+}\right)=\lim _{x \rightarrow 0^{+}} \frac{f_{4}^{\prime}(x)}{g_{4}^{\prime}(x)}=\frac{1}{3}, \quad \varphi_{4}(\infty)=0 .
$$

Therefore, Lemma 6 follows easily from (32), (33), (35), and Lemma 1 together with the monotonicity of $f_{4}^{\prime}(x) / g_{4}^{\prime}(x)$.

Lemma 7. The function

$$
\varphi_{5}(x)=\frac{\log ((x+\sinh (x) \cosh (x)) /(2 \sinh (x)))}{\log [\cosh (x)]}
$$

is strictly increasing from $(0, \log (2+\sqrt{3}))$ onto $(1 / 3$, $[2 \log (2 \sqrt{3}+\log (2+\sqrt{3}))-\log 3] /(2 \log 2)-1)$.

Proof. Let $f_{5}(x)=\log [(x+\sinh (x) \cosh (x)) /(2 \sinh (x))]$ and $g_{5}(x)=\log [\cosh (x)]$. Then simple computations lead to

$$
\begin{aligned}
& \varphi_{5}(x)=\frac{f_{5}(x)}{g_{5}(x)}=\frac{f_{5}(x)-f_{5}\left(0^{+}\right)}{g_{5}(x)-g_{5}(0)}, \\
\frac{f_{5}^{\prime}(x)}{g_{5}^{\prime}(x)}= & \frac{\sinh (x) \cosh ^{3}(x)-x \cosh ^{2}(x)}{x \sinh ^{2}(x)+\sinh ^{3}(x) \cosh (x)} \\
= & \frac{\sinh (4 x)+2 \sinh (2 x)-4 x \cosh (2 x)-4 x}{\sinh (4 x)-2 \sinh (2 x)+4 x \cosh (2 x)-4 x} \\
= & \frac{\sum_{n=1}^{\infty}\left(2^{2 n+2}\left(2^{2 n}-2 n\right) /(2 n+1) !\right) x^{2 n+1}}{\sum_{n=1}^{\infty}\left(2^{2 n+2}\left(2^{2 n}+2 n\right) /(2 n+1) !\right) x^{2 n+1}} \\
= & \frac{\sum_{n=0}^{\infty}\left(2^{2 n+4}\left[2^{2 n+2}-(2 n+2)\right] /(2 n+3) !\right) x^{2 n}}{\sum_{n=0}^{\infty}\left(2^{2 n+4}\left[2^{2 n+2}+(2 n+2)\right] /(2 n+3) !\right) x^{2 n}} .
\end{aligned}
$$

Let

$$
\begin{aligned}
& a_{n}=\frac{2^{2 n+4}\left[2^{2 n+2}-(2 n+2)\right]}{(2 n+3) !}, \\
& b_{n}=\frac{2^{2 n+4}\left[2^{2 n+2}+(2 n+2)\right]}{(2 n+3) !} .
\end{aligned}
$$

Then

$$
\begin{gathered}
a_{n}>0, \quad b_{n}>0, \\
\frac{a_{n+1}}{b_{n+1}}-\frac{a_{n}}{b_{n}}=\frac{(3 n+2) 2^{2 n+2}}{\left(2^{2 n+1}+n+1\right)\left(2^{2 n+3}+n+2\right)}>0
\end{gathered}
$$

for all $n \geq 0$.

It follows from Lemma 2 and (38)-(40) that $f_{5}^{\prime}(x) / g_{5}^{\prime}(x)$ is strictly increasing on $(0, \log (2+\sqrt{3}))$.
Note that

$$
\begin{gathered}
\varphi_{5}\left(0^{+}\right)=\frac{a_{0}}{b_{0}}=\frac{1}{3} \\
\varphi_{5}[\log (2+\sqrt{3})]=\frac{2 \log [2 \sqrt{3}+\log (2+\sqrt{3})]-\log 3}{2 \log 2}-1 .
\end{gathered}
$$

Therefore, Lemma 7 follows from Lemma 1, (37), and (41) together with the monotonicity of $f_{5}^{\prime}(x) / g_{5}^{\prime}(x)$.

Lemma 8. The function

$$
\varphi_{6}(x)=\frac{\log [(x+\sin (x) \cos (x)) /(2 \sin (x) \cos (x))]}{\log [\sec (x)]}
$$

is strictly increasing from $(0, \pi / 3)$ onto $(2 / 3,[2 \log (4 \pi+3 \sqrt{3})-$ $2 \log 6-\log 3] /(2 \log 2))$.

Proof. Let $f_{6}(x)=\log [(x+\sin (x) \cos (x)) /(2 \sin (x) \cos (x))]$, $g_{6}(x)=\log [\sec (x)], f_{7}(x)=\sin (x) \cos (x)-x \cos (2 x)$, and $g_{7}(x)=(x+\sin (x) \cos (x)) \sin ^{2}(x)$. Then simple computations lead to

$$
\begin{gathered}
\varphi_{6}(x)=\frac{f_{6}(x)}{g_{6}(x)}=\frac{f_{6}(x)-f_{6}\left(0^{+}\right)}{g_{6}(x)-g_{6}(0)}, \\
\frac{f_{6}^{\prime}(x)}{g_{6}^{\prime}(x)}=\frac{f_{7}(x)}{g_{7}(x)}=\frac{f_{7}(x)-f_{7}(0)}{g_{7}(x)-g_{7}(0)}, \\
\frac{f_{7}^{\prime}(x)}{g_{7}^{\prime}(x)}=\frac{1}{1 / 2+\sin (2 x) / 2 x} .
\end{gathered}
$$

Since the function $x \rightarrow \sin (2 x) /(2 x)$ is strictly decreasing on $(0, \pi / 3)$, hence Lemma 1 and $(43)$ lead to the conclusion that $\varphi_{6}(x)$ is strictly increasing on $(0, \pi / 3)$.

Note that

$$
\begin{gathered}
\varphi_{6}\left(0^{+}\right)=\lim _{x \rightarrow 0^{+}} \frac{f_{7}^{\prime}(x)}{g_{7}^{\prime}(x)}=\frac{2}{3}, \\
\varphi_{6}\left(\frac{\pi}{3}\right)=\frac{2 \log (4 \pi+3 \sqrt{3})-2 \log 6-\log 3}{2 \log 2} .
\end{gathered}
$$

Therefore, Lemma 8 follows easily from (44) and (45) together with the monotonicity of $\varphi_{6}(x)$. 


\section{Main Results}

Theorem 9. The double inequalities

$$
\begin{aligned}
& A^{\alpha_{1}}(a, b) H^{1-\alpha_{1}}(a, b) \\
& \quad<N_{A H}(a, b)<A^{\beta_{1}}(a, b) H^{1-\beta_{1}}(a, b), \\
& A^{\alpha_{2}}(a, b) H^{1-\alpha_{2}}(a, b) \\
& \quad<N_{H A}(a, b)<A^{\beta_{2}}(a, b) H^{1-\beta_{2}}(a, b), \\
& C^{\alpha_{3}}(a, b) A^{1-\alpha_{3}}(a, b) \\
& \quad<N_{C A}(a, b)<C^{\beta_{3}}(a, b) A^{1-\beta_{3}}(a, b), \\
& C^{\alpha_{4}}(a, b) A^{1-\alpha_{4}}(a, b) \\
& \quad<N_{A C}(a, b)<C^{\beta_{4}}(a, b) A^{1-\beta_{4}}(a, b)
\end{aligned}
$$

hold for all $a, b>0$ with $a \neq b$ if and only if $\alpha_{1} \leq 1 / 3, \beta_{1} \geq 1$, $\alpha_{2} \leq 2 / 3, \beta_{2} \geq 1, \alpha_{3} \leq 1 / 3, \beta_{3} \geq[2 \log (2 \sqrt{3}+\log (2+\sqrt{3}))-$ $\log 3] /(2 \log 2)-1=0.4648 \ldots, \alpha_{4} \leq 2 / 3$, and $\beta_{4} \geq[2 \log (4 \pi+$ $3 \sqrt{3})-2 \log 6-\log 3] /(2 \log 2)=0.7733 \ldots$.

Proof. Without loss of generality, we assume that $a>b>0$. Let $v=(a-b) /(a+b) \in(0,1), p \in(0, \infty), q \in(0, \pi / 2)$, $r \in(0, \log (2+\sqrt{3}))$, and $s \in(0, \pi / 3)$ be the parameters such that $1 / \cosh (p)=\cos (q)=1-v^{2}, \cosh (r)=\sec (s)=1+v^{2}$. Then (9)-(12) lead to the conclusion that inequalities (46)(49) are, respectively, equivalent to

$$
\begin{aligned}
\alpha_{1} & <1-\frac{\log N_{A H}(a, b)-\log A(a, b)}{\log H(a, b)-\log A(a, b)} \\
& =1-\frac{\log [(p+\sinh (p) \cosh (p)) /(2 \sinh (p) \cosh (p))]}{\log (1 / \cosh (p))} \\
& =1-\varphi_{3}(p)<\beta_{1},
\end{aligned}
$$

$\alpha_{2}<1-\frac{\log N_{H A}(a, b)-\log A(a, b)}{\log H(a, b)-\log A(a, b)}$

$$
\begin{aligned}
& =1-\frac{\log [(q+\sin (q) \cos (q)) /(2 \sin (q))]}{\log [\cos (q)]} \\
& =1-\varphi_{4}(q)<\beta_{2},
\end{aligned}
$$$$
\alpha_{3}<\frac{\log N_{C A}(a, b)-\log A(a, b)}{\log C(a, b)-\log A(a, b)}
$$$$
=\frac{\log ((r+\sinh (r) \cosh (r)) /(2 \sinh (r)))}{\log [\cosh (r)]}
$$$$
=\varphi_{5}(r)<\beta_{3} \text {, }
$$

$$
\begin{aligned}
\alpha_{4} & <\frac{\log N_{A C}(a, b)-\log A(a, b)}{\log C(a, b)-\log A(a, b)} \\
& =\frac{\log [(s+\sin (s) \cos (s)) /(2 \sin (s) \cos (s))]}{\log [\sec (s)]} \\
& =\varphi_{6}(s)<\beta_{4} .
\end{aligned}
$$

Therefore, Theorem 9 follows easily from (50)-(53) and Lemmas 5-8.

Theorem 10. Let $\alpha_{5}, \beta_{5}, \alpha_{6}, \beta_{6} \in[0,1 / 2]$. Then the double inequalities,

$$
\begin{aligned}
& H\left[\alpha_{5} a+\left(1-\alpha_{5}\right) b, \alpha_{5} b+\left(1-\alpha_{5}\right) a\right] \\
& \quad<N_{A H}(a, b)<H\left[\beta_{5} a+\left(1-\beta_{5}\right) b, \beta_{5} b+\left(1-\beta_{5}\right) a\right], \\
& H\left[\alpha_{6} a+\left(1-\alpha_{6}\right) b, \alpha_{6} b+\left(1-\alpha_{6}\right) a\right] \\
& \quad<N_{H A}(a, b)<H\left[\beta_{6} a+\left(1-\beta_{6}\right) b, \beta_{6} b+\left(1-\beta_{6}\right) a\right],
\end{aligned}
$$

hold for all $a, b>0$ with $a \neq b$ if and only if $\alpha_{5} \leq 1 / 2-\sqrt{6} / 6=$ $0.09175 \ldots, \beta_{5} \geq 1 / 2-\sqrt{2} / 4=0.1464 \ldots, \alpha_{6} \leq 1 / 2-\sqrt{3} / 6=$ $0.2113 \ldots$, and $\beta_{6} \geq 1 / 2-\sqrt{1-\pi / 4} / 2=0.2683 \ldots$.

Proof. Without loss of generality, we assume that $a>b>0$. Let $\lambda \in[0,1 / 2], v=(a-b) /(a+b) \in(0,1), p \in(0, \infty)$, and $q \in(0, \pi / 2)$ be the parameters such that $1 / \cosh (p)=$ $\cos (q)=1-v^{2}$. Then from (9) and (10) we have

$$
\begin{aligned}
H[ & \lambda a+(1-\lambda) b, \lambda b+(1-\lambda) a]-N_{A H}(a, b) \\
= & A(a, b)\left[1-(1-2 \lambda)^{2} v^{2}\right] \\
& -\frac{1}{2} A(a, b)\left[1+\frac{p}{\sinh (p) \cosh (p)}\right] \\
= & \frac{A(a, b)}{2} \\
& \times\left[1-\frac{2(1-2 \lambda)^{2}(\cosh (p)-1)}{\cosh (p)}-\frac{p}{\sinh (p) \cosh (p)}\right] \\
= & \frac{A(a, b)[\cosh (p)-1]}{2 \cosh (p)} \\
& \times\left[1+\frac{\sinh (p)-p}{\sinh (p)(\cosh (p)-1)}-2(1-2 \lambda)^{2}\right] \\
= & \frac{A(a, b)[\cosh (p)-1]}{2 \cosh (p)}\left[1+\varphi_{1}(p)-2(1-2 \lambda)^{2}\right]
\end{aligned}
$$




$$
\begin{aligned}
H[ & \lambda a+(1-\lambda) b, \lambda b+(1-\lambda) a]-N_{H A}(a, b) \\
= & A(a, b)\left[1-(1-2 \lambda)^{2} v^{2}\right] \\
& \quad-\frac{1}{2} A(a, b)\left[\cos (q)+\frac{q}{\sin (q)}\right] \\
= & \frac{1}{2} A(a, b) \\
& \times\left[2-2(1-2 \lambda)^{2}(1-\cos (q))-\cos (q)-\frac{q}{\sin (q)}\right] \\
= & \frac{1-\cos (q)}{2} A(a, b)\left[1-2(1-2 \lambda)^{2}-\varphi_{2}(q)\right] .
\end{aligned}
$$

Therefore, Theorem 10 follows easily from (55) and (56) together with Lemmas 3 and 4.

Theorem 11. Let $\alpha_{7}, \beta_{7}, \alpha_{8}, \beta_{8} \in[1 / 2,1]$. Then the double inequalities,

$$
\begin{aligned}
& C\left[\alpha_{7} a+\left(1-\alpha_{7}\right) b, \alpha_{7} b+\left(1-\alpha_{7}\right) a\right] \\
& \quad<N_{C A}(a, b)<C\left[\beta_{7} a+\left(1-\beta_{7}\right) b, \beta_{7} b+\left(1-\beta_{7}\right) a\right], \\
& C\left[\alpha_{8} a+\left(1-\alpha_{8}\right) b, \alpha_{8} b+\left(1-\alpha_{8}\right) a\right] \\
& \quad<N_{A C}(a, b)<H\left[\beta_{8} a+\left(1-\beta_{8}\right) b, \beta_{8} b+\left(1-\beta_{8}\right) a\right],
\end{aligned}
$$

hold for all $a, b>0$ with $a \neq b$ if and only if $\alpha_{7} \leq 1 / 2+$ $\sqrt{3} / 6=0.7886 \ldots, \beta_{7} \geq 1 / 2+\sqrt{6 \sqrt{3} \log (2+\sqrt{3})} / 12=$ $0.8082 \ldots, \alpha_{8} \leq 1 / 2+\sqrt{6} / 6=0.9082 \ldots$, and $\beta_{6} \geq 1 / 2+$ $\sqrt{8 \sqrt{3} \pi-18} / 12=0.9210 \ldots$

Proof. Without loss of generality, we assume that $a>b>0$. Let $\mu \in[1 / 2,1], v=(a-b) /(a+b) \in(0,1), r \in(0, \log (2+$ $\sqrt{3}))$, and $s \in(0, \pi / 3)$ be the parameters such that $\cosh (r)=$ $\sec (s)=1+v^{2}$. Then from (11) and (12) one has

$$
\begin{aligned}
C[\mu a+(1-\mu) b, \mu b+(1-\mu) a]-N_{C A}(a, b) \\
=A(a, b)\left[1+(2 \mu-1)^{2} v^{2}\right] \\
\quad-\frac{1}{2} A(a, b)\left[\cosh (r)+\frac{r}{\sinh (r)}\right] \\
=\frac{1}{2} A(a, b) \\
\quad \times\left[2+2(2 \mu-1)^{2}(\cosh (r)-1)-\cosh (r)-\frac{r}{\sinh (r)}\right] \\
=\frac{1}{2} A(a, b)[\cosh (r)-1]\left[2(2 \mu-1)^{2}-1+\varphi_{1}(r)\right],
\end{aligned}
$$

$$
\begin{aligned}
C[\mu a+(1-\mu) b, \mu b+(1-\mu) a]-N_{A C}(a, b) \\
=A(a, b)\left[1+(2 \mu-1)^{2} v^{2}\right] \\
\quad-\frac{1}{2} A(a, b)\left[1+\frac{s}{\sin (s) \cos (s)}\right] \\
=\frac{1}{2} A(a, b) \\
\quad \times\left[1+2(2 \mu-1)^{2}(\sec (s)-1)-\frac{s}{\sin (s) \cos (s)}\right] \\
=\frac{1}{2} A(a, b)[\sec (s)-1]\left[2(2 \mu-1)^{2}-1-\varphi_{2}(s)\right],
\end{aligned}
$$

where the functions $\varphi_{1}$ and $\varphi_{2}$ are defined as in Lemmas 3 and 4 , respectively.

Note that

$$
\begin{aligned}
\varphi_{1}[\log (2+\sqrt{3})] & =1-\frac{\sqrt{3} \log (2+\sqrt{3})}{3}, \\
\varphi_{2}\left(\frac{\pi}{3}\right) & =\frac{4 \sqrt{3} \pi-18}{9} .
\end{aligned}
$$

Therefore, Theorem 11 follows easily from Lemmas 3 and 4 together with (58)-(59).

Theorem 12. Let $S_{A H}(a, b)=S[A(a, b), H(a, b)], S_{H A}(a, b)=$ $S[H(a, b), A(a, b)], S_{A C}(a, b)=S[A(a, b), C(a, b)]$, and $S_{C A}(a, b)=S[C(a, b), A(a, b)]$. Then the inequalities

$$
\begin{aligned}
H(a, b) & <S_{A H}(a, b)<N_{A H}(a, b)<S_{H A}(a, b) \\
& <N_{H A}(a, b)<A(a, b)<M(a, b)<T(a, b) \\
& <S_{C A}(a, b)<N_{C A}(a, b) \\
& <Q(a, b)<S_{A C}(a, b)<N_{A C}(a, b)<C(a, b)
\end{aligned}
$$

hold for all $a, b>0$ with $a \neq b$.

Proof. It follows from $[1,(3.10)]$ and $[11,(4.1)]$ together with $[9,(5)$, Theorems 2 and 5] that

$$
\begin{gathered}
N(a, b)>\frac{a+2 b}{3}>\mathrm{SB}(a, b), \\
H(a, b)<S_{A H}(a, b)<L(a, b)<S_{H A}(a, b)<P(a, b) \\
<A(a, b)<M(a, b)<T(a, b)<S_{C A}(a, b) \\
<Q(a, b)<S_{A C}(a, b)<C(a, b) .
\end{gathered}
$$

Therefore, the second, fourth, ninth, and twelfth inequalities follow from (61) and the first, sixth, seventh, eighth, and eleventh inequalities follow from (62) immediately, while the fifth and thirteenth inequalities can be derived from $H(a, b)<$ $A(a, b)<C(a, b)$ and the fact that $N_{A H}(a, b)$ and $N_{A C}(a, b)$ are, respectively, the mean values of $A(a, b), H(a, b)$, and $A(a, b), C(a, b)$ 
Next, we prove the third and tenth inequalities. In fact, the third inequality can be derived from the following inequalities (63) [14, Theorem 1.2] and (64) [9, Theorem 3] together with $A(a, b)>H(a, b)$. Consider the following:

$$
\begin{gathered}
N_{A H}(a, b)<\frac{1}{2} A(a, b)+\frac{1}{2} H(a, b), \\
S_{H A}(a, b)>\frac{2}{\pi} A(a, b)+\left(1-\frac{2}{\pi}\right) H(a, b),
\end{gathered}
$$

while the tenth inequality can be derived from the inequality $N_{C A}(a, b)<C^{\beta_{3}}(a, b) A^{1-\beta_{3}}(a, b)$ in Theorem 9 and $C(a, b)>$ $A(a, b)$ together with $Q(a, b)=A^{1 / 2}(a, b) C^{1 / 2}(a, b)$, where $\beta_{3}=[2 \log (2 \sqrt{3}+\log (2+\sqrt{3}))-\log 3] /(2 \log 2)-1=$ $0.4648 \ldots$.

Remark 13. He et al. [14, Theorem 1.2], Xia and Chu [17, Theorem 3.1], and Chu et al. [18, Theorem 2.1] proved that the double inequalities

$$
\begin{aligned}
& \alpha_{1} A(a, b)+\left(1-\alpha_{1}\right) H(a, b) \\
& \quad<N_{A H}(a, b)<\beta_{1} A(a, b)+\left(1-\beta_{1}\right) H(a, b), \\
& \alpha_{2} A(a, b)+\left(1-\alpha_{2}\right) H(a, b) \\
& \quad<N_{H A}(a, b)<\beta_{2} A(a, b)+\left(1-\beta_{2}\right) H(a, b), \\
& \alpha_{3} A(a, b)+\left(1-\alpha_{3}\right) H(a, b) \\
& \quad<L(a, b)<\beta_{3} A(a, b)+\left(1-\beta_{3}\right) H(a, b), \\
& \alpha_{4} A(a, b)+\left(1-\alpha_{4}\right) H(a, b) \\
& \quad<P(a, b)<\beta_{4} A(a, b)+\left(1-\beta_{4}\right) H(a, b)
\end{aligned}
$$

hold for all $a, b>0$ with $a \neq b$ if and only if $\alpha_{1} \leq 1 / 3, \beta_{1} \geq$ $1 / 2, \alpha_{2} \leq 2 / 3, \beta_{2} \geq \pi / 4=0.7853 \ldots, \alpha_{3} \leq 0, \beta_{3} \geq 2 / 3$, $\alpha_{4} \leq 2 / \pi=0.6366 \ldots$, and $\beta_{4} \geq 5 / 6$.

From the above results we clearly see that the mean values $N_{A H}(a, b)$ and $L(a, b)$ and $N_{H A}(a, b)$ and $P(a, b)$ are not comparable with each other.

\section{Conflict of Interests}

The authors declare that there is no conflict of interests regarding the publication of this paper.

\section{Acknowledgments}

This research was supported by the Natural Science Foundation of China under Grants 61374086, 11371125, and 11171307 and the Natural Science Foundation of Zhejiang Province under Grant LY13A010004.

\section{References}

[1] E. Neuman and J. Sándor, "On the Schwab-Borchardt mean," Mathematica Pannonica, vol. 14, no. 2, pp. 253-266, 2003.

[2] E. Neuman and J. Sándor, "On the Schwab-Borchardt mean II," Mathematica Pannonica, vol. 17, no. 1, pp. 49-59, 2006.
[3] E. Neuman, "Inequalities for the Schwab-Borchardt mean and their applications," Journal of Mathematical Inequalities, vol. 5, no. 4, pp. 601-609, 2011.

[4] B. C. Carlson, Special Functions of Applied Mathematics, Academic Press, New York, NY, USA, 1977.

[5] B. C. Carlson, "Algorithms involving arithmetic and geometric means," The American Mathematical Monthly, vol. 78, pp. 496$505,1971$.

[6] J. L. Brenner and B. C. Carlson, "Homogeneous mean values: weights and asymptotics," Journal of Mathematical Analysis and Applications, vol. 123, no. 1, pp. 265-280, 1987.

[7] Z.-Y. He, Y.-M. Chu, and M.-K. Wang, "Optimal bounds for Neuman means in terms of harmonic and contraharmonic means," Journal of Applied Mathematics, vol. 2013, Article ID 807623, 4 pages, 2013.

[8] Y.-M. Chu and W.-M. Qian, "Refinements of bounds for Neuman means," Abstract and Applied Analysis, vol. 2014, Article ID 354132, 8 pages, 2014.

[9] E. Neuman, "On some means derived from the SchwabBorchardt mean," Journal of Mathematical Inequalities, vol. 8, no. 1, pp. 171-183, 2014.

[10] E. Neuman, "On some means derived from the SchwabBorchardt mean II," Journal of Mathematical Inequalities, vol. 8, no. 2, pp. 361-370, 2014.

[11] E. Neuman, “On a new bivariate mean," Aequationes Mathematicae, 2013.

[12] Y. Zhang, Y.-M. Chu, and Y.-L. Jiang, "Sharp geometric mean bounds for Neuman means," Abstract and Applied Analysis, vol. 2014, Article ID 949815, 6 pages, 2014.

[13] Z.-J. Guo, Y. Zhang, Y.-M. Chu, and Y.-Q. Song, "Sharp bounds for Neuman means in terms of geometric, arithmetic and quadratic means," In press, http://arxiv.org/abs/1405.4384.

[14] Z.-Y. He, Y.-M. Chu, Y.-Q. Song, and X.-J. Tao, "Note on certain inequalities for Neuman means," http://arxiv.org/abs/ 1405.4387.

[15] G. D. Anderson, S.-L. Qiu, M. K. Vamanamurthy, and M. Vuorinen, "Generalized elliptic integrals and modular equations," Pacific Journal of Mathematics, vol. 192, no. 1, pp. 1-37, 2000.

[16] S. Simić and M. Vuorinen, "Landen inequalities for zerobalanced hypergeometric functions," Abstract and Applied Analysis, vol. 2012, Article ID 932061, 11 pages, 2012.

[17] W.-F. Xia and Y.-M. Chu, "Optimal inequalities related to the logarithmic, identric, arithmetic and harmonic means," Revue d'Analyse Numérique et de Théorie de l'Approximation, vol. 39, no. 4, pp. 176-183, 2010.

[18] Y.-M. Chu, Y.-F. Qiu, M.-K. Wang, and G.-D. Wang, "The optimal convex combination bounds of arithmetic and harmonic means for the Seiffert's mean," Journal of Inequalities and Applications, vol. 2010, Article ID 436457, 7 pages, 2010. 


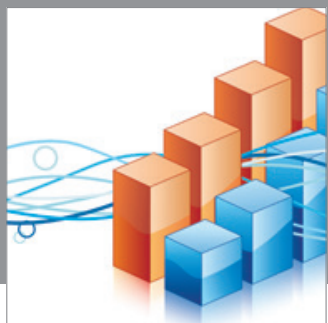

Advances in

Operations Research

mansans

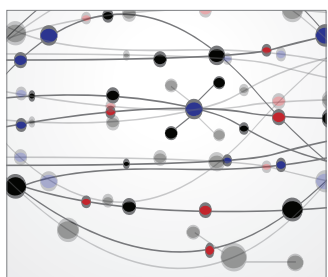

The Scientific World Journal
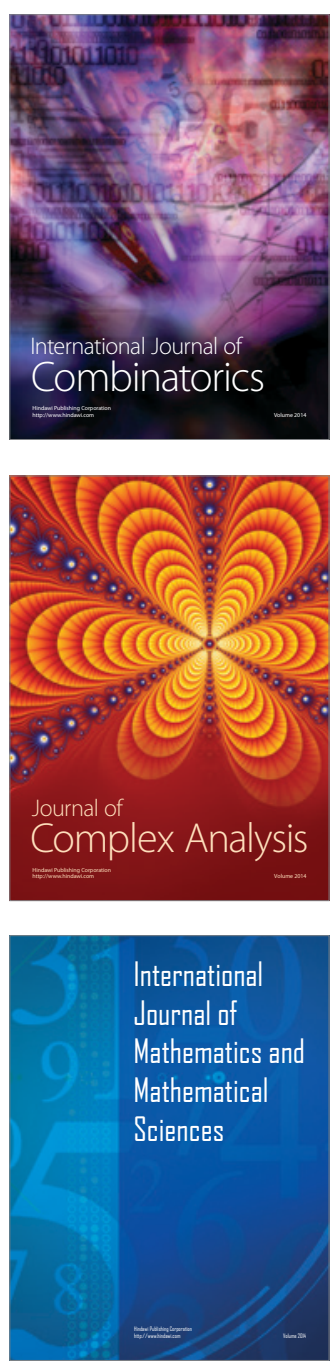
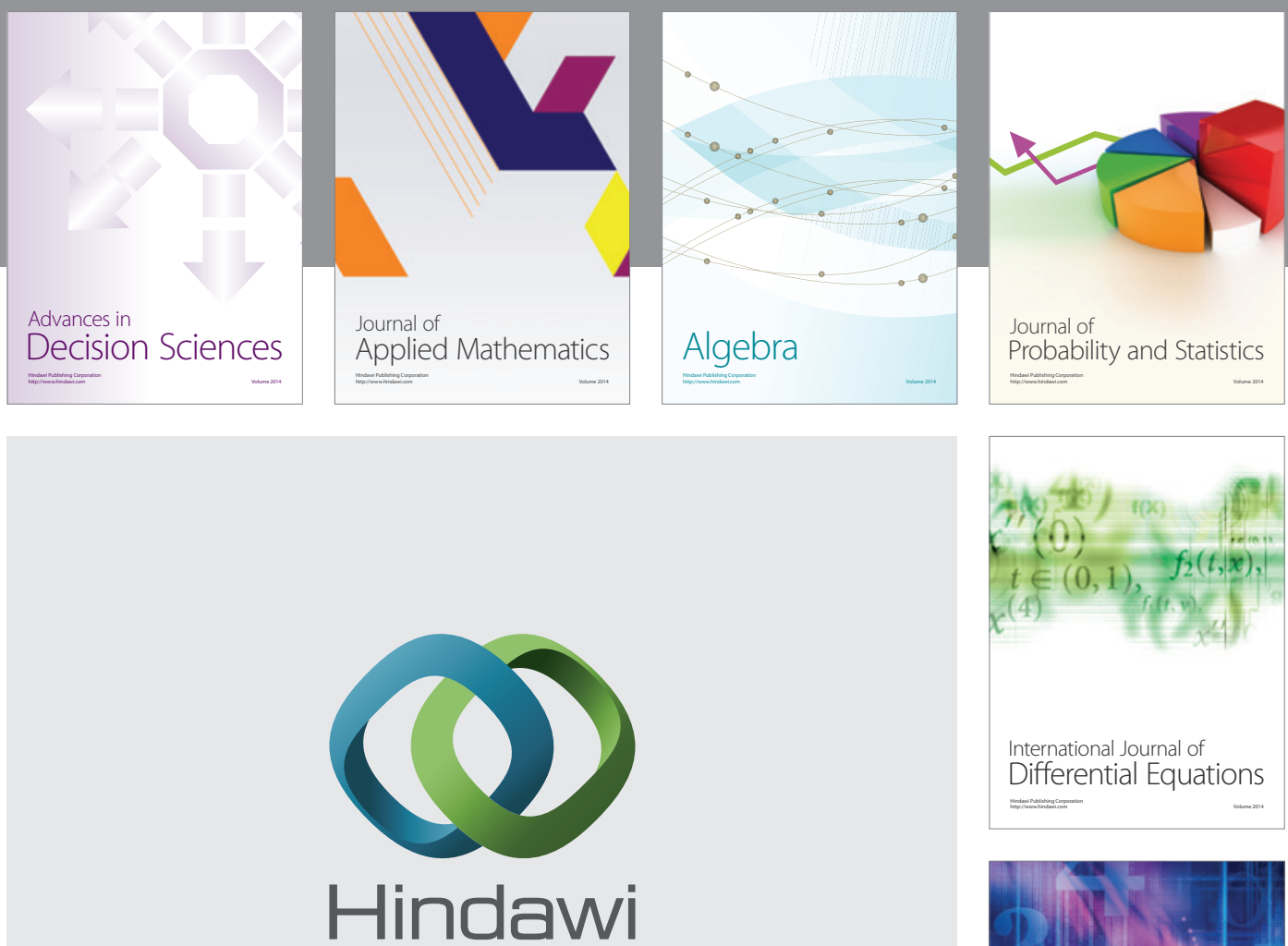

Submit your manuscripts at http://www.hindawi.com
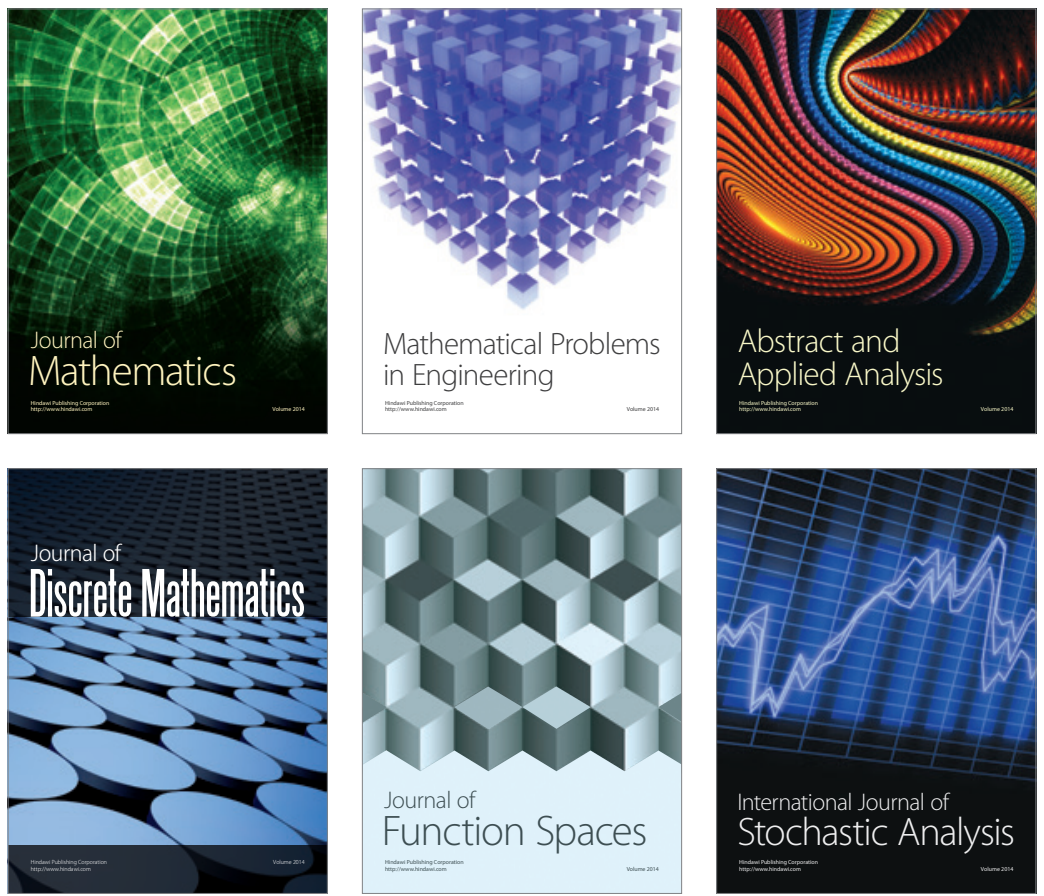

Journal of

Function Spaces

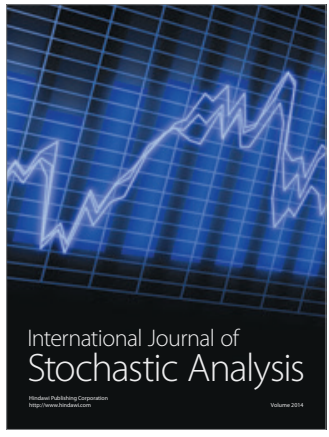

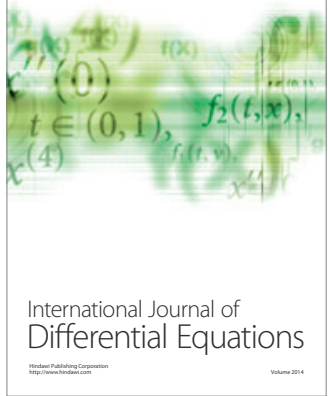
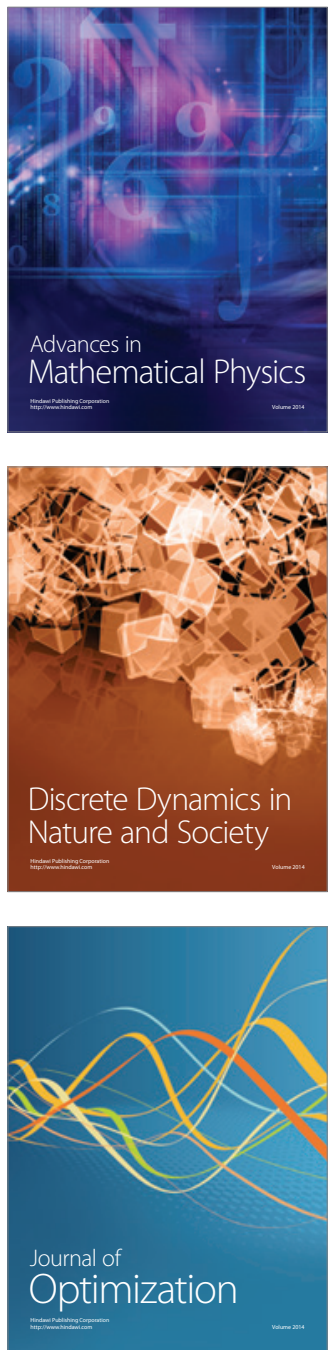